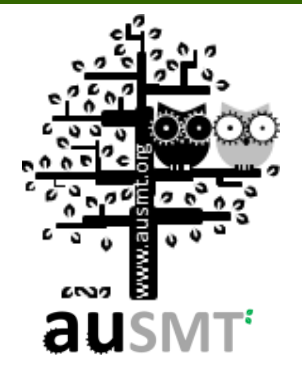

\title{
Automatic Calibration System for Precision Angle Measurement Devices
}

\author{
Yung-Cheng Wang1,*, Lih-Horng Shyu ${ }^{2}$, Eberhard Manske ${ }^{3}$, \\ Chung-Ping Chang ${ }^{1}$, and Shih-Syun Lin ${ }^{1}$ \\ ${ }^{1}$ Department of Mechanical Engineering, National Yunlin University of Science and Technology, Taiwan \\ ${ }^{2}$ Institute of Electro-Optical and Materials Science, National Formosa University, Taiwan \\ ${ }^{3}$ Institute of Process Measurement and Sensor Technology, Chair of the Department of Precision Metrology, Germany \\ (Received 28 January 2014; Accepted 13 March 2014; Published on line 1 September 2014) \\ *Email: wangyc@yuntech.edu.tw \\ DOI: $10.5875 /$ ausmt.v4i3.515
}

Abstract: An optomechatronic module is proposed to achieve automatic and efficient calibration of a compact autocollimators for high precision angle measurement. Conventional autocollimators have several disadvantages including large size and high cost, thus efforts over the past decade have focused on producing compact autocollimators. To satisfy the needs of practical applications, the measuring performance of a compact autocollimator must be determined, raising the need for a reliable and efficient calibration module.

Integration of the photoelectronic sensor, mechanism design and self-developed software produces a process for efficient calibration. The employed sensor is a dual axis position sensitive detector. The system includes an angular sensing module and an angular control mechanism. and signal processing is achieved through a self-developed measuring program. Comparison with a commercial autocollimator indicates that the proposed system can serve as a reference standard to calibrate other compact autocollimators such as DVD pick-ups. The corresponding measurement curve of a calibrated compact autocollimators can then be automatically determined by the measurement program. Experimental results verify that the standard deviation of the DVD pick-up yaw angle is less than 1.5 arcsec and that of the pitch angle is less than $4.5 \operatorname{arcsec}(2 \sigma)$.

With the proposed automatic calibration system, the measuring characteristics of a calibrated compact autocollimator can be determined in a few minutes thus producing low-cost, high-precision angle measurements without commercial autocollimators.

Keywords: Precision angle measurement; automatic calibration; compact autocollimator

\section{Introduction}

Autocollimators are widely used to conduct precision measurements of various mechanical parameters, e.g. tilt angles, straightness and rotary indexing [1-3]. Using a conventional autocollimator, [4-6] angle measurements are performed with manual observations that unavoidably result in operation errors. Optoelectronic autocollimators use optoelectronic sensors to conduct measurements automatically thus enhancing measurement precision and efficiency.

However, conventional optoelectronic autocollimators are still too bulky and expensive for certain applications, including on-line automatic inspections. Compact autocollimators [7-9] have been developed to overcome these issues.

Prior to using compact autocollimators for practical applications, they must be calibrated to determine the correlation between the electronic signals and the corresponding angle measurements. A self-developed automatic calibration system is proposed for this purpose to ensure reduced costs, high efficiency and high measurement precision. 


\section{Measurement Principle}

\section{Optical structure of an autocollimator}

Figure 1 illustrates the measurement principle of a laser autocollimator. When the measuring mirror is tilted with an angle $\theta$, the reflected beam will be rotated with the angle $2 \theta$, resulting in a displacement $\mathrm{d}$ of the focused spot on the optoelectronic detector. The relationship between the tilt angle and the spot displacement can be described by Equation(1), where $f$ is the known focal length of the convex lens. By detecting $d$, the tilt angle can be determined.

$$
d=f \times \tan (2 \theta)
$$

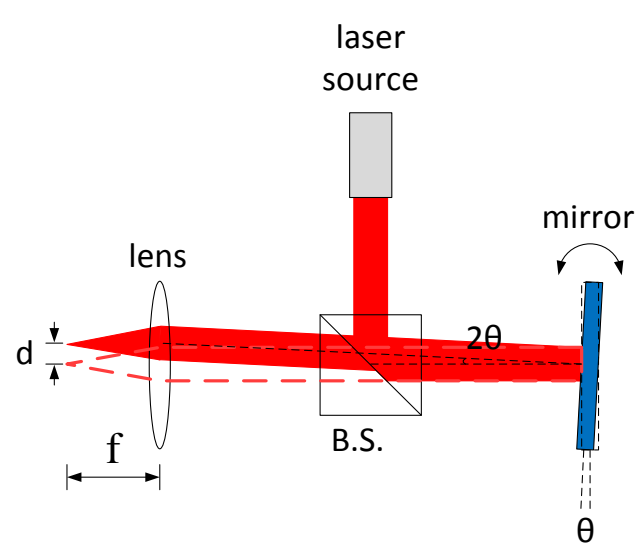

Figure 1. Measurement principle of a laser autocollimator.

\footnotetext{
Yung-Cheng Wang received his Dr.-Ing. from the Institute of Process Measurement and Sensor Technology at the IImenau University of Technology (TU IImenau) in 2003. He is now an associate professor in the Department of Mechanical Engineering at the National Yunlin University of Science and Technology (Yuntech). His research interests include optical metrology and its applications in precision mechanical engineering, and the development of Fabry-Perot inetrefrometers and inspection technologies for machine tools and on-line automated measurements. He has published over 50 research papers and holds 30 patents.

Lih-Horng Shyu is an associate professor in the Department of Electro-optical Engineering of National Formosa University in Taiwan. His research interests include interferometry, confocal metrology, electro-optical system design , and signal processing.

Eberhard Manske holds a foundation professorship in "Precision Measurement Technology" at the IImenau University of Technology, and serves as spokesman for the collaborative research centre "SFB 622 Nanopositioning and Nanomeasuring Machines". His research focuses mainly on nanopositioning and nanomeasuring technology, fibre-coupled laser interferometry, laser stabilization, optical and tactile precision sensors and scanning probe techniques.

Chung-Ping Chang is a research assistant in the Department of Mechanical Engineering of National Yunlin University of Science and Technology in Taiwan. His research focuses on the design of interferometer systems for displacement measurement.

Shih-Syun Lin graduated from the National Yuline University of Science in technology where he reesarched system development for precision angular measurements.
}

\section{Signal sensing component}

In the self-developed module, a two-dimensional Position Sensitive Detector (PSD, DL16-7PCBA3) is used to sense the displacement of the beam spot induced by the tilt angle. From PSD, the relationship between the output signals $\Delta X_{P S D} / \Delta Y_{P S D}$ and the tilt angle $\alpha_{P S D} / \beta_{P S D}$ (i.e., the yaw/pitch angle) is expressed as Equations (2) and (3). Here $K_{x 1} / K_{y 1}$ is the linear coefficient obtained by the calibration procedure and $X_{\text {sum }}$ denotes the voltage sum of $X$-axis on PSD, $Y_{\text {sum }}$ the voltage sum of $Y$-axis.

$$
\begin{gathered}
\alpha_{\mathrm{PSD}}=\mathrm{K}_{\mathrm{x} 1} \frac{\Delta \mathrm{X}_{\mathrm{PSD}}}{\mathrm{X}_{\text {sum }}} . \\
\beta_{\mathrm{PSD}}=\mathrm{K}_{\mathrm{y} 1} \frac{\Delta \mathrm{Y}_{\mathrm{PSD}}}{\mathrm{Y}_{\text {sum }}} .
\end{gathered}
$$

In the compact autocollimator (DVD pick-up), a quadrant photodiode (QD) is used as the sensing component (Figure 2). Four quadrant detection elements in QD serve to sense the intensity variation of the beam spot on the QD induced by the tilt angles. Initially the beam spot is restricted to the QD symmetrically. When there is a tilt angle, the intensity distribution of the reflected beam spot will become unsymmetrical. The correlation between the tilt angle and the intensity distributions on the four elements $A, B$, $C$ and $D$ can be expressed as Equations (4) and (5). Here $\alpha_{P S D} / \beta_{P S D}$ denotes the tilt angle, $K_{x 2} / K_{y}$ the linear coefficient and $V_{A}, V_{B}, V_{C}$, and $V_{D}$ the output voltage of each sensing element.

$$
\begin{aligned}
& \alpha_{D V D}=\mathrm{K}_{\mathrm{x}} \frac{\left(\mathrm{V}_{\mathrm{A}}+V_{\mathrm{C}}\right)-\left(\mathrm{V}_{\mathrm{B}}+V_{\mathrm{D}}\right)}{\mathrm{V}_{\mathrm{A}}+V_{\mathrm{B}}+V_{\mathrm{C}}+V_{D}} . \\
& \beta_{D V D}=\mathrm{K}_{\mathrm{y}} \frac{\left(\mathrm{V}_{\mathrm{A}}+V_{\mathrm{B}}\right)-\left(\mathrm{V}_{\mathrm{C}}+V_{\mathrm{D}}\right)}{\mathrm{V}_{\mathrm{A}}+V_{\mathrm{B}}+V_{\mathrm{C}}+V_{\mathrm{D}}} .
\end{aligned}
$$

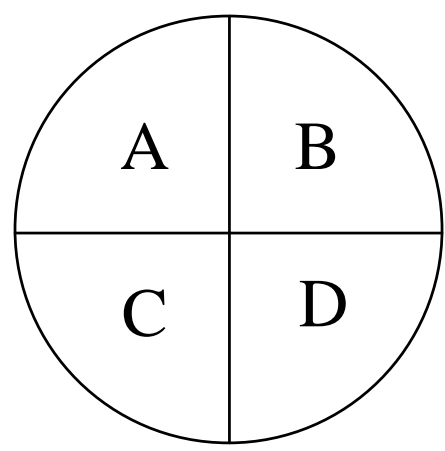

Figure 2. QD sensing elements. 


\section{Structure of Calibration System}

Figure 3 shows the structure of the automatic calibration system and the angular control mechanism. The automatic calibration system includes the self-fabricated autocollimator, a mechanism to regulate the tilt angle and a self-developed measuring program to automatically perform the calibration procedure. Three piezo translators are installed on the fixed platform. The tilt angle $\phi_{x}$ can be regulated using $\mathrm{Pt}_{\mathrm{y} 1}$ and $\mathrm{Pt}_{\mathrm{y} 2}$. Similarly $\phi_{x}$ can be controlled by $\mathrm{PT}_{\mathrm{x}}$ (Figures 4 and 5). A plane mirror is mounted on the movable platform such that it can be tilted by various displacements of the three piezo transducers. The total volume of this module is less than $100 \times 100 \times 100 \mathrm{~mm}^{3}$. Previous experiments have shown that, during the test process, tilt angle errors will not exceed 0.2" [10].

As shown in Figure 3 , the self-developed autocollimator is calibrated using a commercial digital autocollimator (Equipment Solutions model AC424). Measurements made with the self-developed autocollimator and the compact autocollimator (DVD pick-up) were then compared. Following this procedure, the calibration data of the compact autocollimator can be obtained automatically and with the analysis of data fitting the corresponding linear function of the compact autocollimator can be finally determined.

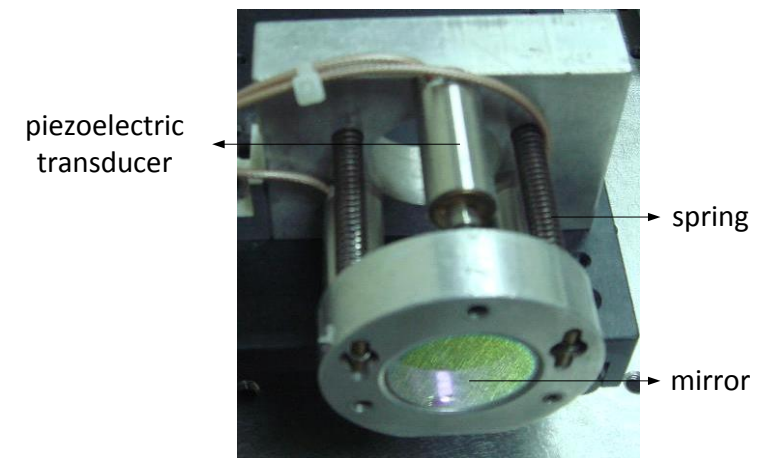

Figure 3. Automatic calibration system with a commercial autocollimator.

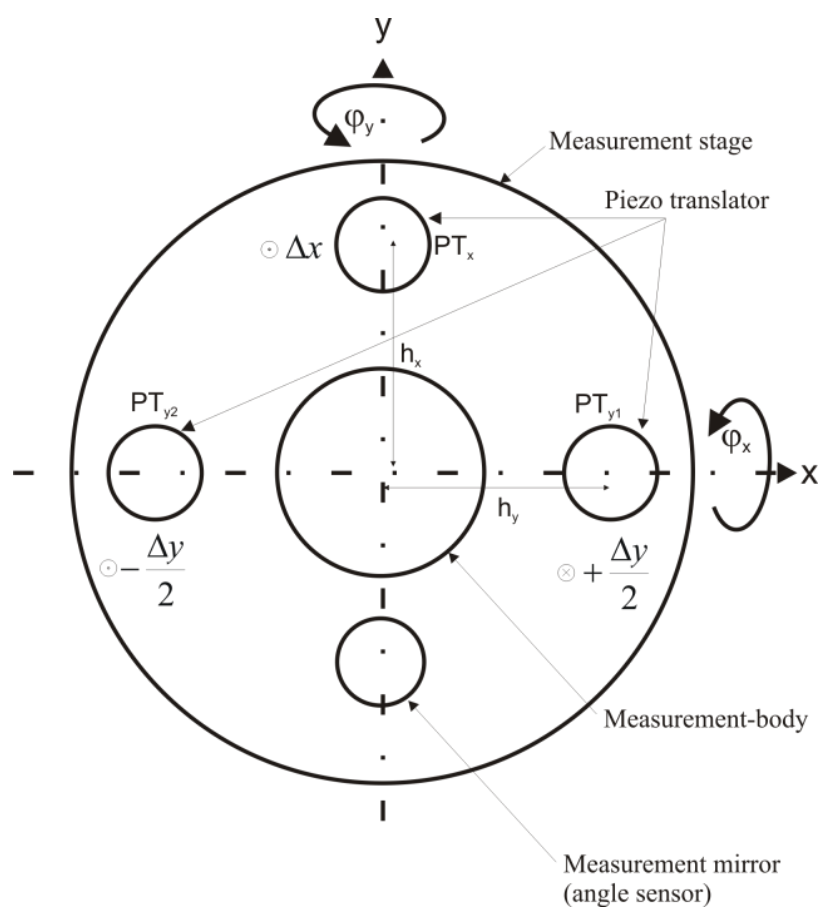

Figure 5. Relationship between piezo-transducers and tilt angles.

Figure 7 diagrams the algorithm for comparing measurements between the reference standard and the calibrated autocollimator. In the measuring program, there are two types of measuring operations to be selected, one for the self-developed autocollimator calibrated with the commercial digital autocollimator (left side) and the other for the compact autocollimator (DVD-pick-up) compared with the self-developed autocollimator (right side) (see Figure 8).

Given 20 measuring points and 10 measurands gained at each point, the entire measurement process takes less than five minutes, resulting in improved calibration efficiency.

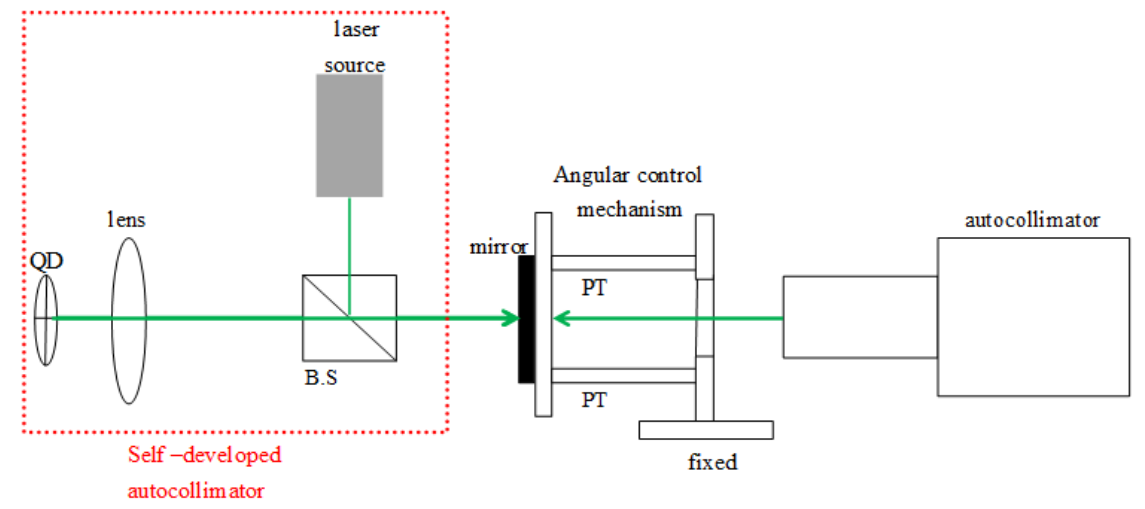

Figure 4. Illustration of angular control mechanism. 


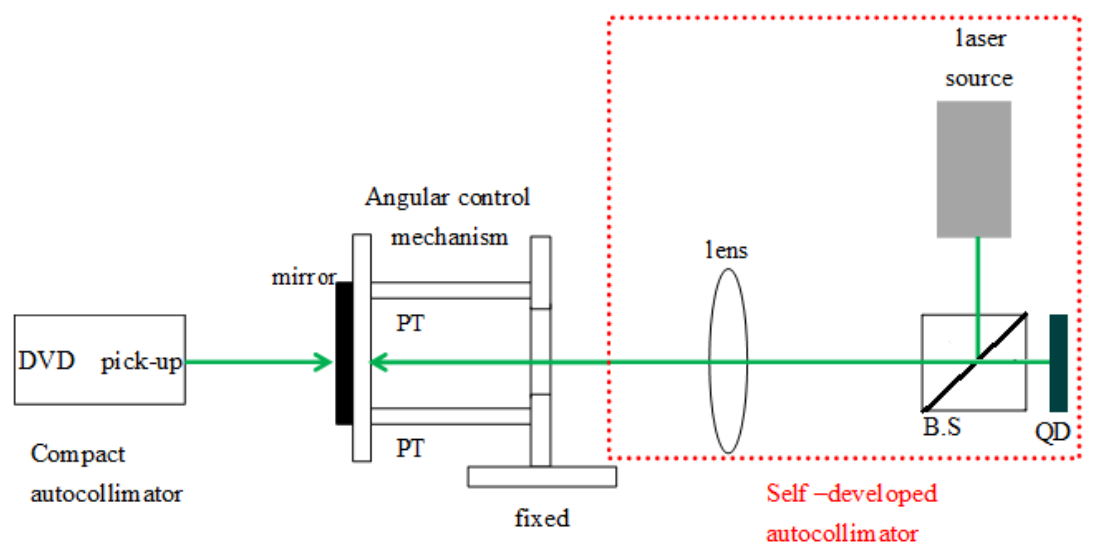

Figure 6. Automatic calibration system with the self-developed autocollimator.

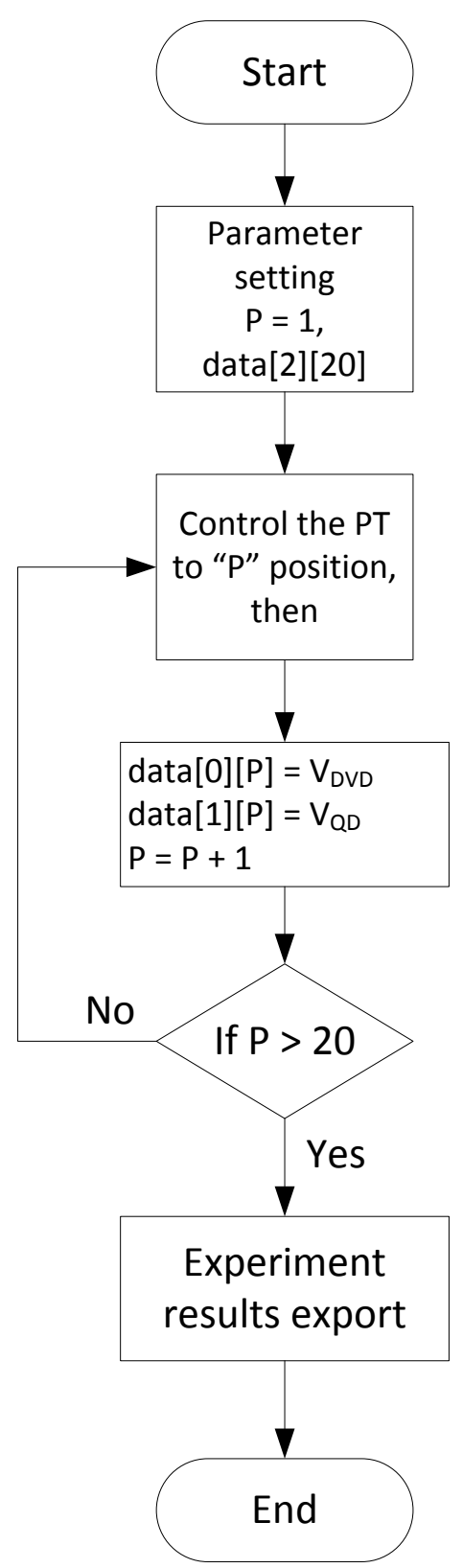

Figure 7. Measurement comparison procedure.

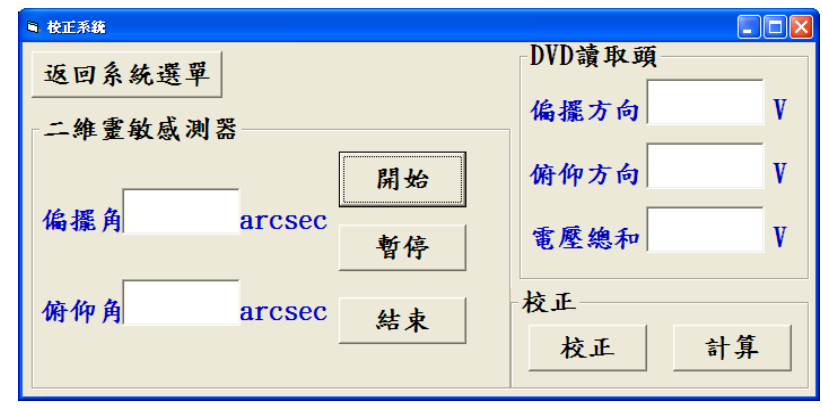

Figure 8. Measuring program interface.

\section{Results and Analysis}

As described above, the tilt angle of the mirror is automatically regulated by the angular control mechanism and the measuring program. The calibration experiments reveal that the measuring performance of the self-developed autocollimator is approximately linear with a maximal linearity error of less than 2 arcsec in the range of \pm 100 arcsec (Figures 9 and 10). This indicates that the measurement results of the self-developed autocollimator are satisfactory and it can be used for secondary calibration.

After the compact autocollimator (DVD pick-up) has been calibrated with the self-developed autocollimator, the function curve of the DVD pick-up is also linear (Figure 11), with the corresponding deviations of the linearity error shown in Figure 12. Experimental verification analyses indicate the standard deviation of yaw angle of the DVD pick-up is less than 1.5 arcsec and that of the pitch angle is less than $4.5 \operatorname{arcsec}(2 \sigma)$. These results indicate that the compact autocollimator can be used for precision angle measurements with measurement requirements below 5 arcsec, making it highly useful for applications characterized by online or minimal volume conditions. 


\section{Conclusion}

This paper proposes an automatic calibration system and related mechanisms for high precision angle measurement. The key findings are as follows:

1. The robustness of the automatic calibration system is verified through a comparison of measurement results with precision compact autocollimators.

2. Experimental testing indicates the standard deviation for the yaw angle of a DVD pick-up is less than 1.5 arcsec and that of the pitch angle is less than $4.5 \operatorname{arcsec}(2 \sigma)$. Thus the proposed system can provide measuring precision of less than 5 arcsec.

3. The available measuring range of the proposed autocollimator is \pm 100 arcsec.

The proposed calibration system provides a high degree of precision and efficiency for the rapid verification of ordinary compact autocollimators. Its performance has been also verified by means of measurement comparison with a compact autocollimator.

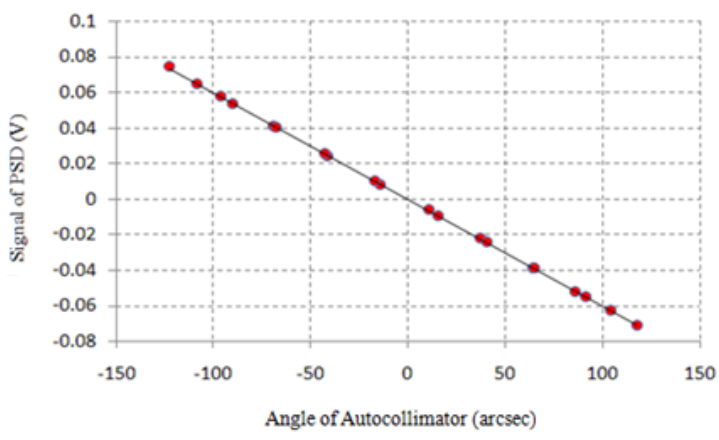

Figure 9. Calibration of yaw angles.

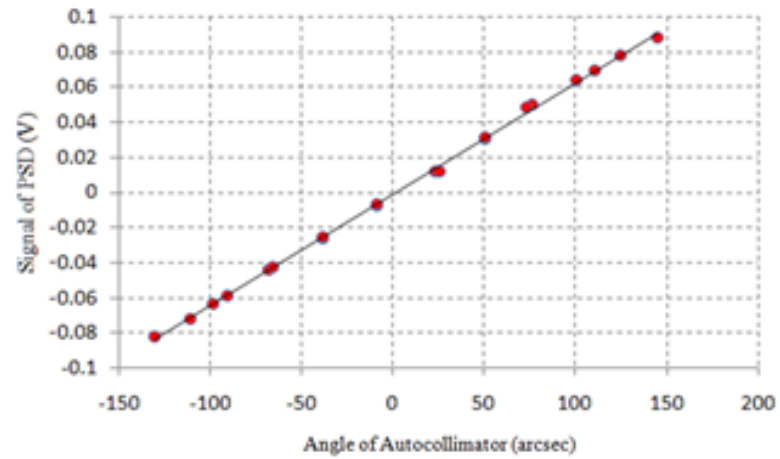

Figure 10. Calibration of pitch angles.

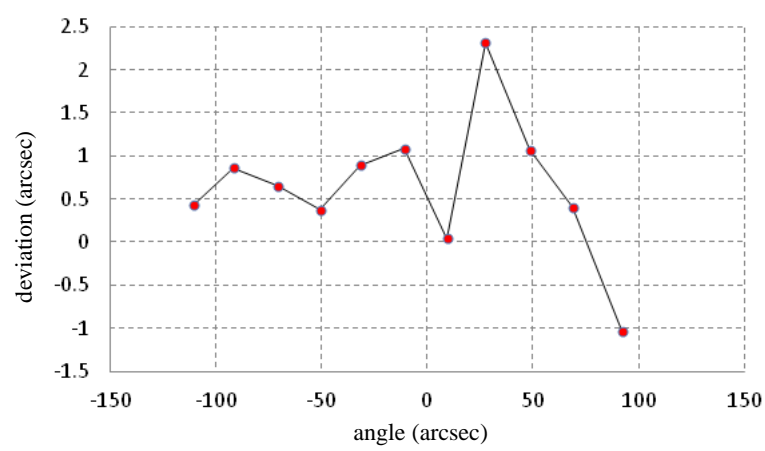

Figure 11. Deviation of yaw angle of the calibrated DVD pick-up

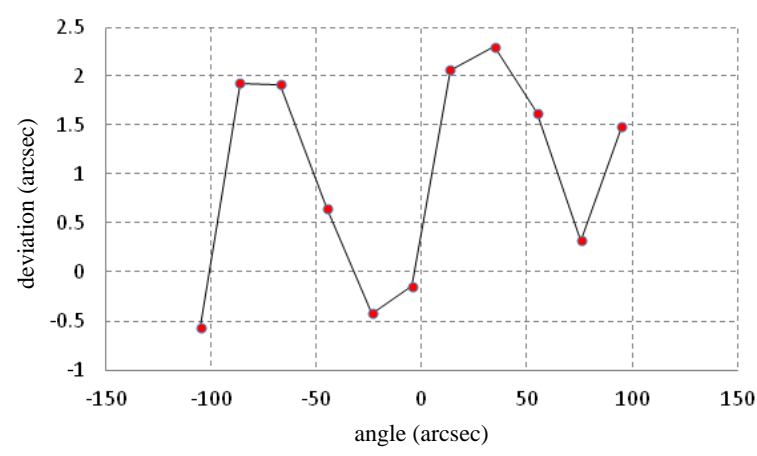

Figure 12. Deviation of pitch angle of the calibrated DVD pick-up.

\section{Acklowledgment}

Authors would like to thank Taiwan's National Science Council for the funding support (grant number NSC 99-2815-C-224-004-E).

\section{References}

[1] M. Welter, E. Manske, and G. Jaeger, "Interferometric sensor and calibration system for high-precision applications," in Proceeding SPIE 5384, San Diego, California, USA, 2004, pp. 26-37. doi: $\underline{10.1117 / 12.538913}$

[2] R. Liu, X. Guo, J. Yu, Y. Qin, Q. Bian, and W. Li, "A novel instrument for high precision angle measurement of optical axes," Optik - International Journal for Light and Electron Optics, vol. 124, no. 12, pp. 1132-1135, 2013.

doi: http://dx.doi.org/10.1016/j.ijleo.2012.03.019

[3] Z. Q. Yin and S. Y. Li, "High accuracy error separation technique for on-machine measuring straightness," Precision Engineering, vol. 30, no. 2, pp. 192-200, 2006.

doi: 10.1016/i.precisioneng.2005.07.006

[4] F. E. Wright, "A new autocollimator," Journal of the Optical Society of America, vol. 9, no. 2, pp. 187-188, 1924. doi: $10.1364 /$ JOSA.9.000187 
[5] M. W. GmbH, Electronic autocollimators, [Online]. Available:

http://www.moeller-wedel-optical.com/en/produk te/electronic-autocollimators.html

[6] A. N. Korolev, A. I. Gartsuev, G. S. Polishchuk, and V. P. Tregub, "A digital autocollimator," Journal of Optical Technology, vol. 76, no. 10, pp. 624-628, 2009.

doi: $10.1364 /$ JOT.76.000624

[7] T. R. Armstrong and M. P. Fitzgerald, "An autocollimator based on the laser head of a compact disc player," Measurement Science and Technology, vol. 3, no. 11, pp. 1072-1076, 1992. doi: $10.1088 / 0957-0233 / 3 / 11 / 009$
[8] W. Gao, "Laser autocollimator for measurement of multi-axis tilt motion," in Precision nanometrology. Springer, 2010, pp. 35-67. doi: 10.1007/978-1-84996-254-4 2

[9] W. Gao, Y. Saito, H. Muto, Y. Arai, and Y. Shimizu, "A three-axis autocollimator for detection of angular error motions of a precision stage," CIRP Annals Manufacturing Technology, vol. 60, no. 1, pp. 515-518, 2011. doi: $10.1016 /$ j.cirp.2011.03.052

[10] Y. C. Wang, W. Y. Jywe, and C. H. Liu, "Development of an automatic test equipment for nano gauging displacement transducers," Journal of Physics: Conference Series, vol. 13, pp. 216-219, 2005. doi: 10.1088/1742-6596/13/1/050 